replaced with no fear of injury or of insufficient force being exerted. This will be appreciated when, for example, an anastomosis by Carrel's method has been completed and it is desired to test the connection before finally allowing the blood to pass from vessel to vessel. It is very true that it does not take long to do this with the Crile clamp, but the shortening of an operation even by seconrls is to be desired, and, whereas in replacing the Crile clamp in a hurry an inexperienced operator may apply too much pressure, this can not be done with the modified clamp if properly adjusted in the first place.

Euclid Avenue and Fast One Hundred and Fifth Street.

\section{A CASE OF BILATERAL BEZOLD MASTOIDITIS FOLLOWING ANGINOUS SCARLET FEVERS.*}

\section{ADOLPH O. PFINGST, M.D.} LOUISVILLE, KY.

The following case presents so many clinical features of interest that a full report seems justifiable:

Bistory.-When I first saw this child the following history was obtained from the family physician: A girl, aged 11, previously healthy, began, on the third or fourth day, after the onset of a well-developed scarlatinal eruption, to show signs of involvement of the nasal, pharyngeal and faucial mucous membranes. Several days later the patient began to complain of pain in her left ear and on the following day both ears were discharging pus.

Examination.-There was marked involvement of the mucous membrane of the upper respiratory tract, especially of the nose. The turbinates were swollen, blocking the nares. They were bathed in a yellowish pus, which had caused an excoriation of the nostrils and skin beneath. A stringy muco-pus could also be seen on the posterior pharyngeal wall. Both faucial tonsils were enlarged and partially covered with a thin grayish-yellow deposit. A peculiar disagreeable odor was noticeable, emanat. ing from the mouth and nose of the child. There was no glandular enlargement at the time. A rather thick pus was discharging from both ears through large openings in the posterio-inferior quadrant of the drums. There was no swelling of the ear canal nor over the mastoid and no tenderness over the bone. Temperature 99.5 to $100.5 \mathrm{~F}$. The urine was normal. Owing to the obstruction, nasal respiration was interfered with. The enforced mouth breathing added to the discomfort of the child by causing a dryness of the buccal mucous membrane. There was some pain and considerable difficulty in deglutition.

Treatment.-The mucous membranes of the nose and throat were treated by spraying with a warm carbolic solution, the nasal passages being previously opened by the use of cocain. The tonsils were swabbed with a solution of potassium permangante and potassium chlorate, and the ears were irrigated every two hours with a warm saline solution under low pressure. The ehild continued to have from one to one and a half degrees of fever. She grew weaker and more emaciated daily, but showed no other symptoms, except the enlargement of the lymphatic glands of the neck. In two weeks the swelling had reached considerable proportions. Three weeks after the beginning of the otorrhea, the patient complained for the first time of pain in and behind the right ear, the left one being unchanged. She had been growing more irritable and resented any effort to manipulate the glandular mass. Pressure over either bone did not seem to elicit pain. There was slight swelling just at the tip of the mastoid which was apparently part of the general swelling of the neck. There was no swelling over the region of the mastoid antrum, and no sagging of the superior pasterior wall of the external auditory canal. On the following day, however, there was decided swelling over the 907.

* Read before the Jefferson County Medical Society, Feb. 15, lower end of the bone, so that an operation on the right side was decided on.

Operation.-The conditions found were interesting. No yus was present external to the bone. The antrum was rather large and was filled with foul pus. After cleansing this and removing the neighboring diseased cells and curetting the aditus, I was about to dress the wound when, with the searcher, a small rough area was detected low down on the median side of the bone cavity. The removal of this area led to several apparently healthy cells and their removal uncovered another pus cavity. When this was thoroughly exposed by the removal of overhanging ledges of bone, a small opening was found leading into the digastric fossa, from which a small quantity of pus exuded when pressure was made below the tip. The entire tip was removed and the case dressed in the usual manner. Although the only symptoms of mastoid involvement noted on the left side were slight swelling and questionable tenderness, An exploratory operation was decided on. Conditions prevailed almost identical with those on the other side. Pus had not perforated the outer table of the bone. The rather large and superficial antrum was full of pus and communicated through several smaller diseased cells with a smaller pus cavity. This in turn was found to communicate by a small opening with the digastric fossa. The mastoid tip was removed entirely on this side also and the wound treated as it was on the riglit side. Both wounds closed in seven and one-half weeks and the drums were restored. The hearing, two months later, had returned to almost normal and the child is the picture of health, the glands in the neck having almost disappeared.

This case exemplifies the destructive nature of middleear abscesses complicating scarlet fever of the anginous variety. The other feature of interest was the development of the mastoid abscess with few or no symptoms. It would be impossible to determine how long the perforation into the digastric fossa had existed before the operation. The swelling and tenderness along the sternomastoid muscle characteristic of Bezold mastoiditis were entirely obscured by the marked glandular swelling in the neck. It is rather infrequent that the mastoid operation is demanded on both sides at once and a unique condition that both had perforated into the digastric fossa, especially in childhood when the antrum is superficial and situated high up.

\section{A HOSPITAL SIGNAL SYSTEM.}

$$
\begin{aligned}
& \text { P. C. H. PAHL, B.S., M.D. } \\
& \text { LOS ANGELES, CAL. }
\end{aligned}
$$

In October, 1904, I devised, for the Hospital of the Good Samaritan, a signal system for calling nurses to patients, which has the following advantages:

1. Noiseless. There being no bells, the call is absolutely silent.

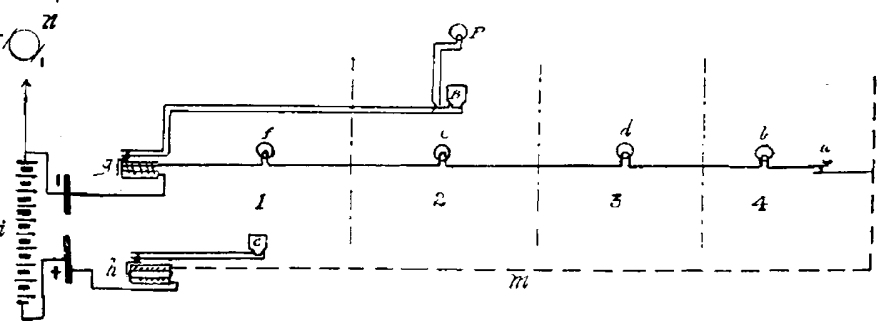

Fig. 1.-Diagram of wiring, signal system, Good Samaritan Hospital, Los Angeies.

2. Monitor board. This is in the office of the superintendent of nurses and enables this officer or her representatives to observe each call for the entire institution, thus making it possible to see at a glance the number of patients desiring the services of a nurse and the length of time elapsing before such call is answered. 
3. Lights in the corridors. These are placed above the door of each patient's room, making it possible for the nurse, if she happens to be in the vicinity, to observe and answer the call without having to go to the indicator in the nurse's room.

4. Lights in the rooms. These are placed in such a manner that they may be readily observed by the patients, who may thus know, when attempting to signal for a nurse, whether they have been successful or not.

5. Specially constructed switches. These are placed in the rooms in connection with the lights and are operated by means of two cords. One of these cords passes through lollow moulding substituted for ordinary picture moulding, and leaves the moulding at the side of the bed, being so arranged as to be readily grasped by the patient who pulls it, thus closing the switch and putting on the signals. The other cord is placed in such a posi-
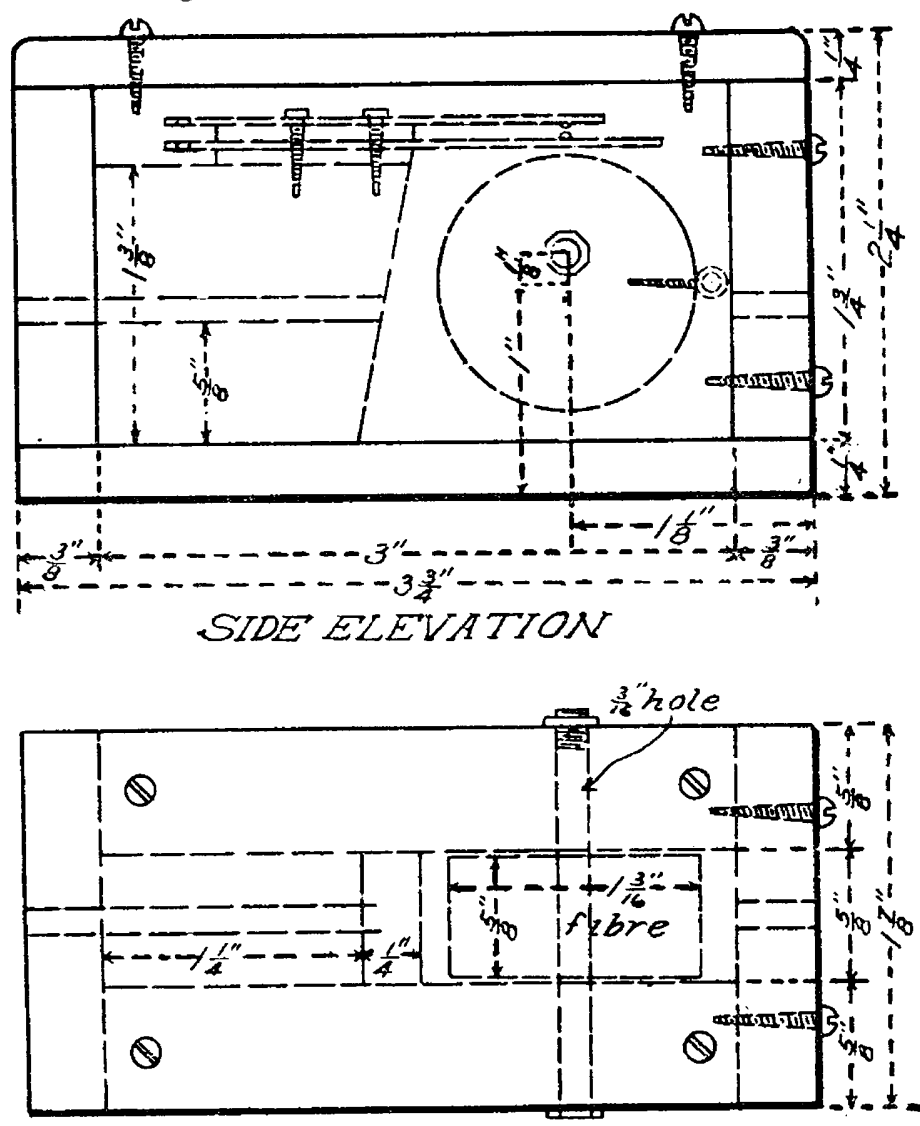

\section{PLAN}

Fig. 2.--Switch for signal system.

tion that, while it is impossible for the patient to reach it from the bed, it is easily accessible to the nurse as she enters the room. Pulling this cord restores the switch and indicates that the call has been answered.

6. Signal boards. The hospital is divided into five distinct departments, each under the charge of a head nurse who has her headquarters in a nurse's room. In each of these rooms is placed a signal board which contains the number of each patient's room in that department, so that only those calls are registered.

\%. Pilot lights. Each department has its subkitchen in which is placed a pilot light which lights with every call in that department, thus enabling the night nurse, who spends considerable time in the subkitchen preparing diets, to observe when some one is signaling. On the ceiling at the intersection of the cross hall is placed mother pilot light which lights simultaneously with the one in the subkitchen; this light is visible from any point in the halls of an entire department.

Figure 1 is a diagram of the wiring; $a$ represents the switch (Fig. 2) located in the patient's room; $b$ the light in the patient's room; $d$, the light in the corridor ; $e$, the light on the signal board in the nurse's room; $f$, the light on the monitor board in the office of the superintendent; $g$ is a relay in the common return to the signal board in the nurse rooms. Each time a call is made in that department this works buzzer $B$ and pilot light $P$ in multiple with it. $h$, located on the monitor board, is a relay in the common return of the entire system and causes buzzer $c$ to operate when a call is made from any room in the entire hospital. The buzzers $B$ and $c$ are ordinarily not used, the pilot lights and the lights on the monitor board being sufficient. $m$ is the common return and $n$ the source of energy, which may be anything over fifty volts and which charges the storage battery $i$.

Figure 2 represents a specially constructed switch, the body of which is made of wood in which the two terminals are platinum points mounted on two German silver springs. The contact is made by causing a fiber cylinder to revolve on an eccentric of one-eighth of an inch.

This system has been in operation over two years and has proved to be a great help in giving prompt, efficient and noiscless service. Concerning the source of energy, if direct current exists in a hospital, the storage battery may be charged through a bank of incandescent lights; if only alternating current is available, however, it is necessary to install a small motor generating set with which to charge the batteries.

934 West Seventh Street.

\section{'THE INELUENCE OF ALCOHOL AND CHLORO- FORM ON PHAGOCYTOSIS IN VITRO.*}

\section{GEORGE RUBIN, M.D. chicago.}

In a previous communication ${ }^{2} I$ have shown how the natural defenses of the animals employed in the experiments were lowered or suspended when subjected to thorough narcotization. In the several series of experiments it was noted that the narcotized animals reacted poorly to various infections artificially produced. It was also shown that the recovery or death of the rabbits depended apparently on the condition of the leucocytes. Narcotized rabbits when infected showed a hypoleucocytosis of various degrees, whereas the controls usually showed a pronounced leucocytosis which was followed by complete recovery or at least survival of their narcotized fellows by a considerable space of time.

These phenomena suggested the possibility that the narcotics had a deterimental influence on the phagocytic function of the leucocytes. It was also shown that there is a more active intraperitoneal phagocytosis of carmine granules in normal than in narcotized animals.

It was now thought advisable to study the effect of narcotics on phagocytes in vitro. The technic employed is as follows:

* From the Pathological Laboratory of Rush Medical College, University of Chicago.

* Read before the Chicago Medical Society, March 27, 1907.

1. Influence of Alcobol, Ether and Chloroform on Natural Immunity in Its Relation to Leucocytosis and Phagocytosis, Jour. Infect. Dis., May 30, 1904. 